\title{
Robotic ICSI (Intracytoplasmic Sperm Injection)
}

\author{
Zhe Lu, Member, IEEE, Xuping Zhang, Member, IEEE, Clement Leung, Navid Esfandiari, Robert F. Casper, \\ and Yu Sun*, Senior Member, IEEE
}

\begin{abstract}
This paper is the first report of robotic intracytoplasmic sperm injection (ICSI). ICSI is a clinical procedure performed worldwide in fertility clinics, requiring pick-up of a single sperm and insertion of it into an oocyte (i.e., egg cell). Since its invention 20 years ago, ICSI has been conducted manually by a handful of highly skilled embryologists; however, success rates vary significantly among clinics due to poor reproducibility and inconsistency across operators. We leverage our work in robotic cell injection to realize robotic ICSI and aim ultimately, to standardize how clinical ICSI is performed. This paper presents some of the technical aspects of our robotic ICSI system, including a cell holding device, motion control, and computer vision algorithms. The system performs visual tracking of single sperm, robotic immobilization of sperm, aspiration of sperm with picoliter volume, and insertion of sperm into an oocyte with a high degree of reproducibility. The system requires minimal human involvement (requiring only a few computer mouse clicks), and is human operator skill independent. Using the hamster oocyte-human sperm model in preliminary trials, the robotic system demonstrated a high success rate of $90.0 \%$ and survival rate of $90.7 \%(n=120)$.
\end{abstract}

Index Terms-Automation, cell manipulation, computer vision, ICSI, robotics, sperm manipulation.

\section{INTRODUCTION}

$\mathbf{T}$ HE World Health Organization reports that averagely 8$10 \%$ of couples worldwide experience infertility. Many of these couples require in vitro fertilization (IVF) treatment to conceive. In IVF, intracytoplasmic sperm injection (ICSI) is a procedure in which a single sperm is injected directly into an oocyte (i.e., egg cell) to overcome issues such as male infertility [1].

In the past two decades since its invention, ICSI has been conducted manually by highly skilled embryologists who look into the eyepieces of a microscope while dexterously controlling

Manuscript received February 22, 2011; revised April 11, 2011; accepted April 11, 2011. Date of publication April 25, 2011; date of current version June 17, 2011. The authors acknowledge financial support from the Natural Sciences and Engineering Research Council of Canada and Ontario Centres of Excellence. Asterisk indicates corresponding author.

Z. Lu, X. Zhang, and C. Leung are with the Advanced Micro and Nanosystems Laboratory, University of Toronto, 5 King's College Road, Toronto, ON M5S 3G8 Canada (e-mail: zhe.lu@utoronto.ca; zhxp@mie.utoronto.ca; clement.leung@utoronto.ca).

N. Esfandiari and R. F. Casper are with the Department of Obstetrics and Gynaecology, University of Toronto, Toronto, ON M5S 2X9, Canada (e-mail: nesfand@yahoo.com; casper@lunenfeld.ca).

${ }^{*} \mathrm{Y}$. Sun is with the Advanced Micro and Nanosystems Laboratory, University of Toronto, 5 King's College Road, Toronto, ON M5S 3G8 Canada (e-mail: sun@mie.utoronto.ca).

Color versions of one or more of the figures in this paper are available online at http://ieeexplore.ieee.org.

Digital Object Identifier 10.1109/TBME.2011.2146781 multiple devices (e.g., micromanipulators, pump, microscope stage). However, long training, stringent skill requirements, low success rates from poor reproducibility (e.g., success rates vary from $50 \%$ to $80 \%$ in IVF clinics in North America), and inconsistency among operators in manual operation call for the reduction of human involvement and automated ICSI.

ICSI involves more than cell injection. Before an oocyte is injected, a single sperm must be tracked, immobilized, and aspirated into a sharp injection micropipette. Due to the small size ( $\leq 1 \mu \mathrm{m}$ thickness) and the fast movement $(\geq 25 \mu \mathrm{m} / \mathrm{sec})$ of the sperm tail, the visibility of the sperm tail is rather low under various optical microscopy conditions, making the identification and visual tracking of a moving sperm tail challenging.

Tracking low-contrast objects has long been a topic of interest to the robotics and automation community. Since edge information is often lost or cannot be extracted from low-contrast objects in images, Chan et al. developed an active contour-based object detection algorithm that does not require edge information [2]. However, the method requires numerous iterations before converging to a solution, rendering the algorithm unsuitable for real-time applications. The Kalman filter is optimal for tracking low-contrast objects that exhibit linear and Gaussian temporal dynamics [3]. The sperm tail's movement, however, possesses complex nonlinear and nonGaussian temporal dynamics, making the Kalman filter unsuitable for the sperm tail tracking task.

When a sperm is picked up, the next step is to inject it into an oocyte. The past decade has witnessed significant engineering efforts to automate cell injection using automation and robotics approaches (e.g., [4]-[6]). These systems all borrowed the architecture directly from manual operation and automated only a few steps. Steps that are challenging to a robotic system such as aligning a micropipette relative to a cell, searching for a cell, orienting it, and switching from one cell to the next, were still conducted by a skilled human operator. Robotic research emphases were placed upon precision microrobotic motion control, development of computer vision algorithms for localization and visual tracking of cells and micropipettes, visual servo control of the micropipette, or integration of visual and haptic interfaces.

This paper presents a robotic ICSI system featuring fast oocyte positioning, automated sperm tracking and immobilization, and adaptive oocyte injection with minimal human involvement. A cell holding device was developed to hold many oocytes into a regular pattern. A sperm tail tracking algorithm robust to the low-contrast appearance and the nonlinear fast movement of the sperm tail was developed. A computer vision algorithm was developed to recognize oocyte structures for adapting injection parameters. Experimental results demonstrate that the system is capable of conducting ICSI with high success and survival rates. 


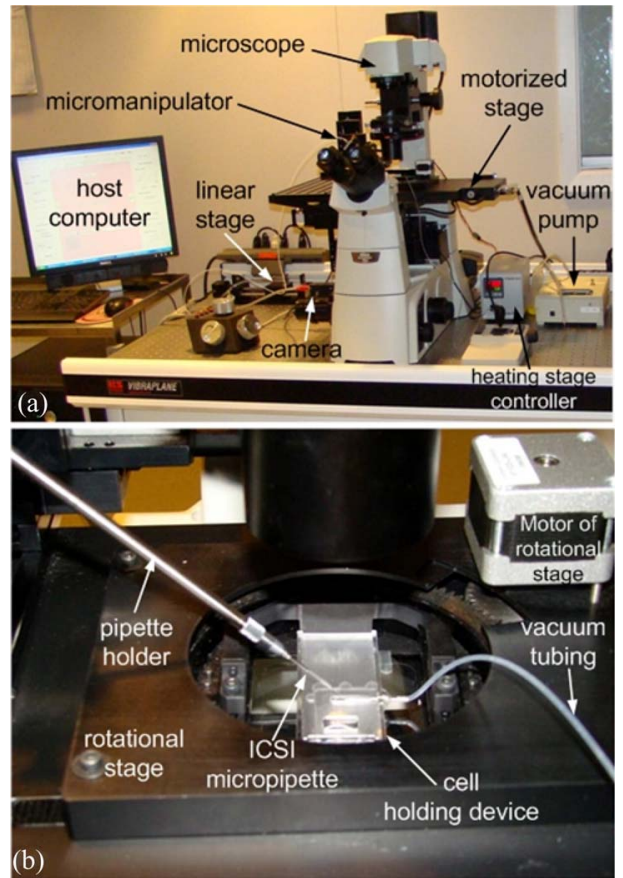

Fig. 1. Robotic ICSI system setup. (a) System picture. (b) Closeup view of the manipulation area.

\section{System SETUP}

As shown in Fig. 1, the robotic ICSI system consists of a standard inverted microscope (Nikon Ti-S), a CMOS camera (601f, Basler), an in-house developed vacuum-based cell holding device for holding multiple oocytes, an in-house developed precision vacuum pump to provide pressure ranging between $-2.5 \mathrm{kPa}$ and $2.5 \mathrm{kPa}$ for holding and releasing oocytes, an in-house developed motorized rotational stage [7] placed on a motorized $\mathrm{X}-\mathrm{Y}$ translational stage (ProScan, Prior Scientific Inc.) for oocyte positioning and orientation control, a straight ICSI micropipette (MIC-50-0, Humagen) connected to a 25 microliter glass syringe (Hamilton), filled with mineral oil, and mounted on a linear stage (eTrack, Newmark System Inc.) for computer-controlled sperm aspiration and deposition, a 3-degrees-of-freedom motorized micromanipulator (MP285, Sutter Inc.) for positioning the ICSI micropipette $\left(30^{\circ}\right.$ tilting angle) to diagonally penetrate oocytes, a heating stage (THN60-10, LINKAM) to maintain oocytes and sperm at $37^{\circ}$ and a host computer for controlling multiple motion control devices and processing images in real time.

Different from conventional ICSI setups that consist of two micromanipulators and use both a holding micropipette and an injection micropipette, our system contains only one micromanipulator and uses a single injection micropipette for the complete ICSI task.

\section{SYSTEM OPERATION}

An operator deposits sperm and oocytes onto the customdeveloped cell holding device [see Fig. 2]. The device consists of an oocyte well with through-holes, a sperm well, a sealed cham-
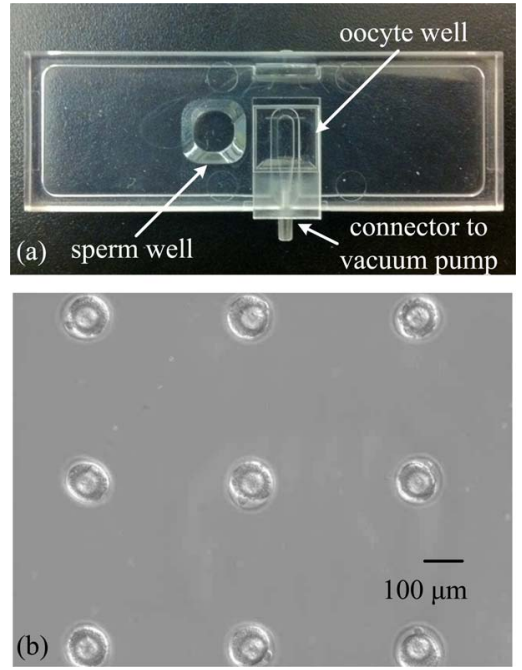

Fig. 2. Cell holding device for ICSI use. (a) Device picture. (b) Picture showing nine oocytes immobilized on the device.

ber, and a connector connecting the chamber to a precision vacuum pump. The sealed chamber is underneath the oocyte well. When oocytes and culture medium are placed into the oocyte well, the oocytes are batch held/released by the application of negative/positive pressure via the sealed chamber. The devices were constructed via injection molding. The through-holes (e.g., $45 \mu \mathrm{m}$ for hamster oocytes) were laser drilled (excimer laser, $193 \mathrm{~nm}$ ) with little debris and highly clean features. Fig. 2(b) shows that nine oocytes are immobilized on the cell holding device, which takes less than one minute. Due to the known positions of oocytes immobilized on the cell holding device, the system is able to readily locate oocytes without conducting random search.

Despite the small size of oocytes and their 2-D appearance under microscopes, an injection micropipette must be 3 -D aligned relative to an oocyte for performing manipulation tasks. Focusdefocus approaches [8] do not offer sufficient accuracy for cell manipulation tasks, and methods reported for contact detection using force or touch sensors (e.g., [9], [10]) complicate system setup. Our system integrates a vision-based contact detection algorithm to automatically determine the vertical position of the micropipette tip and device surface in the oocyte area [11]. The system performs contact detection in both the sperm well and the oocyte well before manipulating sperm and oocytes.

The operator selects a sperm by computer mouse clicking in the proximity of the sperm head. Alternatively, the system is also capable of automatic sperm selection based on sperm locomotive behavior (e.g., speed). The system tracks the motion of the selected sperm and automatically taps its tail for immobilization. A sperm must be immobilized before injection to prevent its interference with the cytoskeleton and metaphase spindle of the oocyte [12]. After the sperm is aspirated into the micropipette [see Fig. 3], the first oocyte is brought into the field of view. If needed, the oocyte is rotated to move the polar body, a cellular structure, away from the penetration site to avoid damage to the spindle [7]. The system performs penetration, 


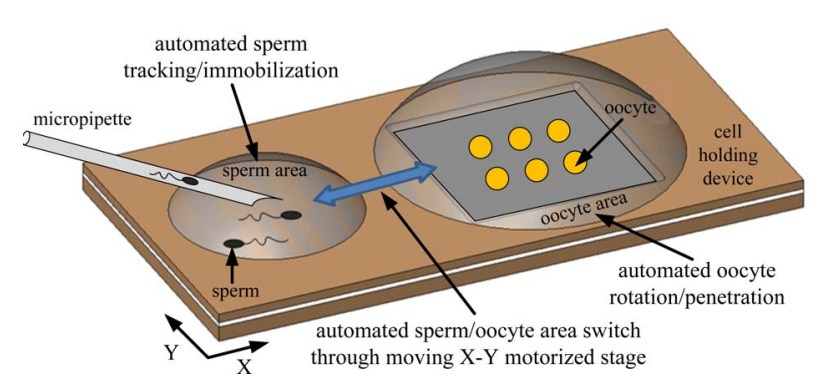

Fig. 3. Robotic ICSI consists of automated sperm manipulation and oocyte injection.

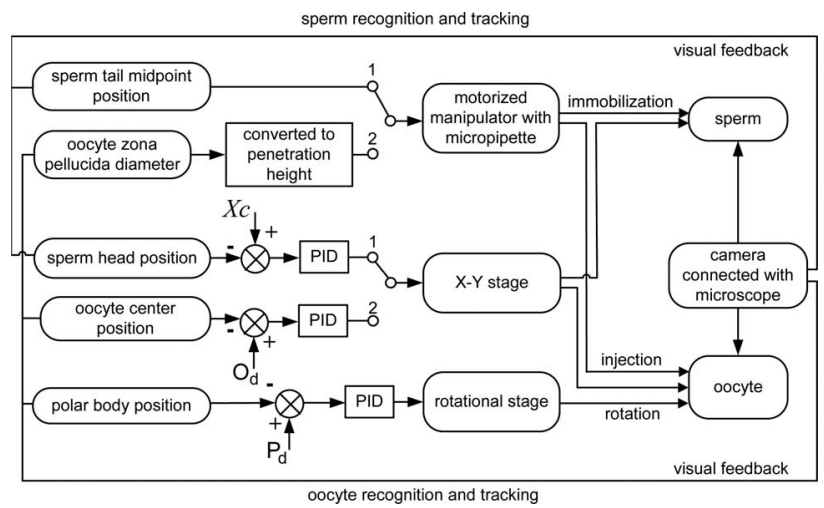

Fig. 4. Control architecture of the robotic ICSI system. Motion control devices include a 3-degrees-of-freedom micromanipulator, a motorized X-Y translational stage, and a motorized rotational stage. Switch 1 is for sperm visual servoing and immobilization. Switch 2 is for oocyte injection.

sperm cell deposition, and micropipette retraction all through computer control. The sperm manipulation and oocyte injection process is repeated until all oocytes are injected.

There are three motion control devices in the system including the micromanipulator, $\mathrm{X}-\mathrm{Y}$ translational stage, and rotational stage that are cooperatively controlled for automated sperm and oocyte manipulation. The overall control architecture of the system is described in Fig. 4. Microscopy visual feedback, which is critical for both sperm and oocyte manipulation, is used for identifying sperm and oocyte structures. Furthermore, visual tracking based on image feedback is used for providing position feedback to guide the operation of the micromanipulator, $\mathrm{X}-\mathrm{Y}$ translational stage and rotational stage. The $\mathrm{X}-\mathrm{Y}$ stage and rotational stage are controlled simultaneously to control the orientation of an oocyte [7]. Details of image-based visual servo control for sperm tracking and immobilization are described in the next section.

\section{Automated SPERM MANipUlation}

Due to the fast movement of healthy sperm, a sperm cell can out of the field of view of a microscope quickly. The low-contrast appearance of the sperm tail also makes the identification of sperm tail challenging. To achieve automated sperm manipulation in robotic ICSI, sperm head and trail tracking algorithm is essential for: 1) visually tracking the spatial location of the sperm, 2) servoing the microscope stage to move the sperm to the center of the field of view, and 3) averaging the tracked head and tail positions to locate the midpoint of the sperm tail for robotic immobilization. Consistently tapping the midpoint of the sperm tail is preferred to prevent damage to the sperm head [13].

Several algorithms have been developed in the field of computer-assisted sperm analysis (CASA) to track sperm trajectories, measure sperm velocity, and evaluate sperm energetics [14], [15]. Prior work for estimating the direction of a sperm tail used the minor and major axes of the sperm head's morphology [16]. The sperm head, however, has a wide variety of shapes [17]. Thus, the approach does not always provide the correct direction of the sperm tail. Despite the considerable progress made in CASA, automated detection and tracking of sperm tails have not been explored.

In our robotic ICSI system, the sperm tracking algorithm first tracks the sperm head to provide visual feedback of the sperm position. Then the sperm tail region of interest (STROI) is extracted. The STROI aims to capture the tail tip region of the sperm. Once the STROI is found, the maximum intensity region (MIR) algorithm is used to locate a point on the sperm tail within the STROI.

\section{A. Sperm Head Tracking and Visual Servoing}

The sperm head is distinctive and, hence, is used for tracking the position of a sperm cell. With the sperm head position, $\mathbf{c}(i)=\left(c_{x}(i), c_{y}(i)\right)$, the spatial distance from the sperm head $\mathbf{c}(i)$ to the center of the field of view $\mathbf{X}_{c}$ is determined. The spatial distance is input into a PID controller, which is used to visually servo the $\mathrm{X}-\mathrm{Y}$ stage to keep the sperm at the center of the field of view [see Fig. 4].

The sperm head tracking process is initiated by a human operator who selects a desired sperm head to track via mouse click on or within proximity to the sperm head. The system obtains a $40 \times 40$ sperm head region of interest (SHROI) from the current frame $i$. The SHROI image's center is initially at the mouse clicked position. The SHROI image is binarized by applying Otsu's adaptive thresholding algorithm. The contour of the sperm head in the SHROI image is computed. The sperm head position $\mathbf{c}(i)$ is found by calculating the moment of the contour. The SHROI is then updated to be a $40 \times 40$ region of interest centered at the sperm head's centroid. For subsequent frames, a similar process is executed to track the sperm head position. However, instead of a mouse click to initiate the process, the SHROI of the previous frame is used by the system as the initial $40 \times 40$ SHROI.

There may be instances in which foreign particles or other sperm enter the SHROI. The sperm head tracking algorithm must be able to differentiate between the sperm of interest and interfering sperm or foreign objects that enter the SHROI. The swimming direction vector of the sperm of interest is used as a unique identifier to discriminate the sperm of interest from other sperm or particles. In the situation where only one sperm is present in the SHROI, the sperm's current direction vector $\mathbf{v}(i)$ in the current frame, represented by (1), is found by subtracting the sperm centroid position in the previous frame $\mathbf{c}(i-1)$ from 


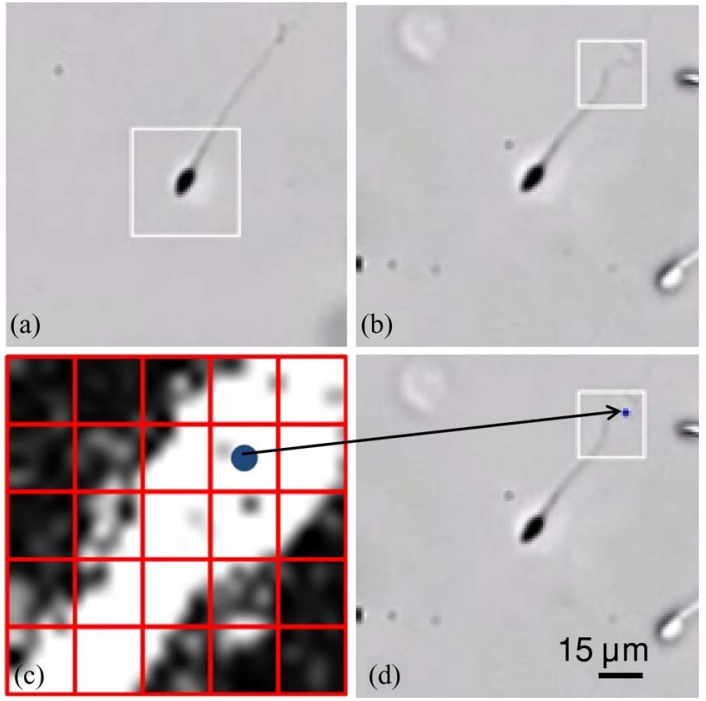

Fig. 5. Steps of the MIR algorithm. (a) A $40 \times 40$ pixels sperm head region of interest (SHROI) is found. (b) A $25 \times 25$ pixels sperm tail region of interest (STROI) is found. (c) Magnification of white box in Fig. 5(b), $5 \times 5$ window (represented by the red boxes) is used to scan and find the $5 \times 5$ section with the highest intensity sum in the flicker image. The center point (blue dot) of the $5 \times 5$ window with the highest intensity sum is considered the tail location. (d) Frame displaying the MIR's estimation of a point on the sperm tail.

the sperm centroid position in the current frame $\mathbf{c}(i)$ :

$$
\mathbf{v}(i)=\mathbf{c}(i)-\mathbf{c}(i-1) .
$$

When more than one sperm or object is present in the SHROI, (1) is extended to

$$
\mathbf{v}(i, s)=\mathbf{c}(i, s)-\mathbf{c}\left(i-1, s_{\text {prev }}\right)
$$

where $s$ represents each sperm in the SHROI, and $s_{\text {prev }}$ is the sperm of interest in the previous frame. The candidate sperm $s$ that produces the minimum Euclidean distance value is considered the sperm of interest $s_{\text {soi }}$ :

$$
s_{\text {soi }}=\min _{s \in[1, N]}\left\{\left\|\mathbf{v}(i, s)-\mathbf{v}\left(i-1, s_{\text {prev }}\right)\right\|\right\}
$$

where $N$ is the total number of sperm and objects inside the SHROI.

\section{B. Sperm Tail Tracking}

With the sperm head position found [Fig. 5(a)], the sperm tail region of interest (STROI), in which the tail is located, can be computed. An example of the STROI is shown in Fig. 5(b). In the ICSI procedure, healthy energetic sperm with strong movement are desired. Hence, our tracking algorithm is only concerned with sperm that are in motion. The average unit direction vector of the sperm movement, and the sperm head position are used to find the STROI. The unit average direction vector is used instead of the direction vector described by (3), because the sperm may exhibit abrupt local changes in movement direction between two consecutive frames. By averaging the direction vectors of the sperm across 30 frames, abrupt changes in the sperm direction between frames are mitigated. Each spatial component of the average direction vector $\overline{\mathbf{v}}(i)$ for a given frame $i$ is found by

$$
\overline{\mathbf{v}}(i)=\frac{1}{30} \sum_{k=0}^{29} c_{r}(i-k)-c_{r}(i-k-1)
$$

where $r$ represents the spatial coordinate values $x$ and $y$. Each spatial component of the unit average vector $\hat{v}_{r}(i)$ can then be calculated to be

$$
\hat{v}_{r}(i)=\frac{\bar{v}_{r}(i)}{\|\overline{\mathbf{v}}(i)\|} .
$$

The STROI's center position $t_{r}(i)$ is found by adding a scaled value of the direction vector to the sperm head's centroid

$$
t_{r}(i)=c_{r}(i)+a \cdot \hat{v}_{r}(i)
$$

where $a$ is a scalar value. Studies have shown that the average human sperm length is approximately $50 \mu \mathrm{m}$ [11]. Under $20 \times$ magnification this value converts to a length of approximately 120 pixels. Experimentally we found that a value $[70,90]$ is appropriate for $a$, as the value compensates for the sperm tail's constant contraction and bending. The STROI is obtained by taking a $25 \times 25$ ROI centered at the $t_{r}(i)$ position. A $25 \times 25$ STROI provides a sufficient tail search area that takes into account a range of different sperm tail lengths and the tail's transversal displacement.

After finding the STROI, the algorithm verifies that a tail is present in the STROI. The fundamental feature of flicker is extracted by taking the absolute difference between six consecutive inverted grayscale image frames

$$
f(i)=\sum_{k=0}^{5}|I(i-k)-I(i-k-1)|
$$

where $f(i)$ is the flicker image extracted at frame $i$, and $I$ represents the grayscale images containing the sperm of interest at frames $i$ to frame $i-5$. Each pixel in the flicker image is squared to enhance the pixel values of areas in which the tail is present. The pixel sum in the STROI of the $f(i)$ image is used as a measurement to determine the presence of a sperm tail. If the pixel sum is above a specific threshold $(\beta=8000)$, a tail is considered to be present. The threshold value $\beta$ was found experimentally by comparing the pixel sum values of STROI images in which a tail exists against cases where no tail exists. Since the flicker image excludes uniform background information, this $\beta$ value can be applied to different microscopic brightness conditions. However, $\beta$ may need to be adjusted to compensate for variations in noise floor conditions across different microscope cameras. An example of the flicker image is shown in Fig. 5(c). If the pixel sum is below the threshold value $\beta$, no tail can be found and the human operator could be informed via a prompt message that the tail has been lost. This situation can happen if the sperm of interest becomes out of focus, resulting in the tail disappearing from the field of view.

Once the sperm tail is confirmed to exist within the STROI, the MIR algorithm is used to detect a point on the sperm tail. The MIR algorithm uses the flicker image. The motivation behind this algorithm is that an individual frame may not show the sperm tail due to the tail's low contrast and fast movement. 


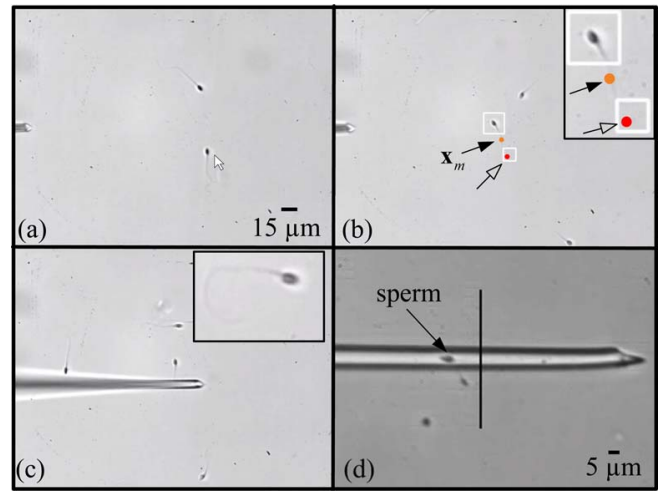

Fig. 6. Sperm immobilization and aspiration operation sequence. (a) Human operator selects a sperm of interest by computer mouse clicking. (b) Sperm head and tail tracking is performed. The black arrow points to the calculated tail midpoint position $\mathbf{x}_{m}$ (orange point) and the hollow arrow points to the calculated tail position (red point). (c) Sperm is tapped and immobilized. (d) Sperm is aspirated into the micropipette.

However, by extracting the flicker feature of the sperm tail [as shown in Fig. 5(c)], the position of the sperm tail can be prominently seen. This approach overcomes challenges that arise from the low-contrast image of the sperm tail in a single frame. The algorithm first finds the location of maximum intensity within the $25 \times 25$ STROI of the flicker image. This is accomplished by evaluating the sum of the intensity values inside a $5 \times 5$ window at a spatial sampling interval of 5 pixels in both the $\mathrm{x}$ and $\mathrm{y}$ coordinates of the STROI flicker image. The center position of the $5 \times 5$ window with the largest intensity value is considered the tail location (i.e., a point on the sperm tail).

\section{Sperm Immobilization}

Successful tracking of sperm head and tail is indispensable for the automation of sperm immobilization. Fig. 6 shows the operation sequence of automated sperm immobilization. The system positions the micropipette at $25 \mu \mathrm{m}$ above the bottom of the sperm well and $100 \mu \mathrm{m}$ left to the center of the field of view [Fig. 6(a)]. An operator selects a sperm of interest by computer mouse clicking on or in proximity to the sperm head [Fig. 6(a)]. This step permits the human operator to select a desired sperm based on sperm morphology and motility, thus exercising his/her expert knowledge. The system then starts to track the sperm head and tail [Fig. 6(b)].

With the sperm head position tracked, the spatial distance from the sperm head to the center of the field of view is determined. The spatial distance is used as the reference input of a PID controller to visually servo the X-Y stage and keep the sperm within the center region of the field of view [see Fig. 4]. Starting values of PID gains were obtained using standard methods, and their final values were determined through experimental trials.

The midpoint of the sperm tail $\mathbf{x}_{m}$ is determined by averaging the tracked head and tail positions. The system controls the micromanipulator to produce a sequence of motions. The midpoint of the sperm tail is tapped by the injection micropipette against the bottom of the sperm well to immobilize the sperm [Fig. 6(c)]. The sperm is then aspirated into the micropipette

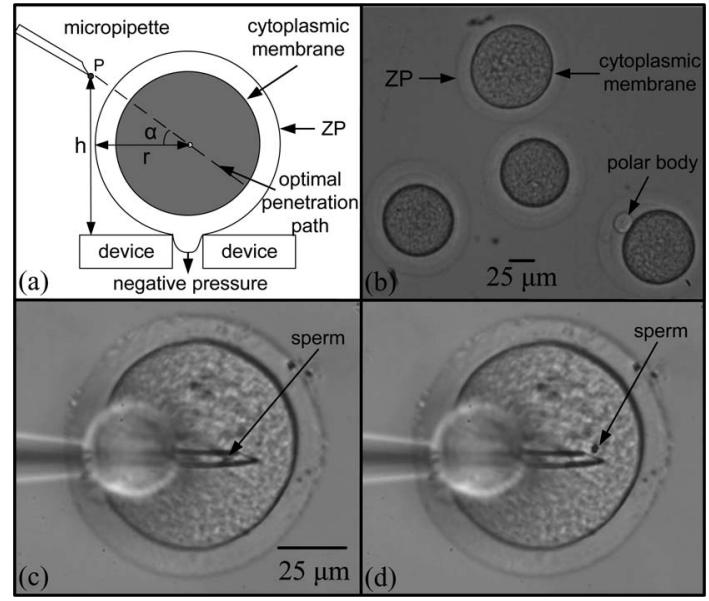

Fig. 7. (a) Oocyte sizes vary significantly, requiring penetration parameter adaptation. (b) A proper penetration height is important for ensuring successful ICSI and a high post-injection survival rate. (c)(d) Injection of an oocyte and sperm deposition. The center circle is from the through-hole on the cell holding device, underneath the oocyte for cell immobilization via vacuum.

from the head and its head is positioned at the black line [Fig. 6(d)].

\section{OOCYTE INJECTION}

Oocyte penetration parameters (e.g., penetration speed, height, and depth) must be well controlled to ensure a high postinjection survival rate. For example, as shown in Fig. 7(a), when the penetration height is too large (above point $\mathrm{P}$ ), the oocyte would rotate because of the generated torque. When the penetration height is too low (below point $\mathrm{P}$ ), the micropipette could collide with the cell holding device or fail to penetrate the zona pellucida [ZP in Fig. 7(a)] and/or the cytoplasmic membrane. A proper penetration height of the micropipette (position $\mathrm{P}$ ) is desired to penetrate each oocyte through the center of the cytoplasmic membrane.

In ICSI, oocyte sizes and their ZP sizes vary significantly [Fig. 7(b)] [18]. As an improvement over our previous cell injection work [19], the robotic ICSI system adaptively determines the penetration height based on the radius of each oocyte's ZP. Considering that the negative pressure only slightly deforms a very small part of the oocyte, the oocyte maintains a shape approximating a sphere [Fig. 7(a)]. Thus, penetration height of the micropipette is $\mathrm{h}=r(1+\tan \alpha)$. Recognition of the cytoplasmic membrane and $\mathrm{ZP}$ is through a series of steps including adaptive thresholding to create a binarized image of the oocyte, calculation of the center of the mass of the binarized image, locating the contour of the cytoplasmic membrane, and determination of the $\mathrm{ZP}$ via iterative growth of the radius of the cytoplasmic membrane. Thus, the system adjusts penetration parameters by calculating the size of an oocyte. Fig. 7(c), (d) shows the injection of an oocyte and sperm deposition.

\section{RESUlTS AND Discussions}

\section{A. Sperm Tracking and Manipulation}

Trials on 100 sperm were first performed to evaluate the system's efficacy for sperm tracking. The system achieved a 
sperm tail tracking success rate of $96 \%$. Sperm tail tracking failed when the sperm enters dark areas of the medium (e.g., air bubbles or large foreign particles). This type of failure can be reduced by manually eliminating bubbles in the culture medium and by ensuring that the culture medium has a low level of contaminants during sample preparation.

The efficacy for sperm immobilization was evaluated on another 1000 sperm. Overall, the system achieved an immobilization success rate of $94.4 \%$ (i.e., 944 out of 1000 sperm were successfully immobilized). The average successful immobilization time was determined to be 6-7 seconds. Most of the failure cases were due to the sudden increase in sperm speed during the sperm tail tapping step. In this step, sperm head and tail tracking is disabled to prevent tracking errors resulting from the occlusion of the sperm by the micropipette [Fig. 6(c)]. Thus, the $\mathrm{X}-\mathrm{Y}$ stage is servoed at the last PID computed velocity. Since the constant velocity of the X-Y stage is not always able to compensate for the sudden increase in sperm speed, the micropipette either misses the sperm tail or hits the very tip of the sperm tail, which often is not sufficient for immobilizing the sperm due to the extreme thinness of the sperm tail tip.

\section{B. Robotic ICSI}

The performance of the robotic ICSI system was quantified by injecting 120 hamster oocytes with donated human sperm. The model of hamster oocyte injection with human sperm is widely used in ICSI training [20]-[22]. The human sperm injected hamster oocytes are not able to develop beyond the two pronucleate stages (i.e., the earliest stage of development) thereby ameliorating ethical concerns. The average time cost in each injection cycle, which includes tracking, immobilization, aspirating a sperm cell and injecting it into an oocyte, was approximately two minutes per ICSI operation. This speed is comparable to highly experienced ICSI operators with over 10 years' experience.

The morphology of injected oocytes were inspected visually immediately after injection. The injected oocytes were then cultured inside at $37^{\circ} \mathrm{C}$ with $5 \% \mathrm{CO}_{2}$. The morphologies of the injected oocytes were checked 20 hours after culture to determine the condition of the oocytes. Oocytes with both clear, continuous cytoplasmic membrane and small periviteline space were considered alive [23].

For quantitative assessment, success rate was defined as the ratio of the number of injected oocytes with sperm successfully deposited in the cytoplasm to the total number of injected oocytes. Survival rate was defined as the ratio of the number of injected oocytes that were alive after 20 hours incubation to the total number of injected oocytes with sperm successfully deposited in the cytoplasm.

Experimental results are summarized in Table I. Of the five independent trials performed by operators having no ICSI training, the robotic ICSI system produced highly consistent results, independent of operator skills (success rate 86.7\%-93.3\%; survival rate $88.5 \%-92.9 \%$ ). The average success rate and survival rate were $90 \%$ and $90.7 \%$. The high survival rate demonstrates that robotic injection did not cause significant damage to cell development. In the low number of cases where sperm deposition
TABLE 1

HAMSTER ICSI RESULTS PRODUCED BY THE ROBOTIC ICSI SYSTEM

\begin{tabular}{|c|c|c|c|c|c|c|}
\hline Experiments & 1 & 2 & 3 & 4 & 5 & Total \\
\hline No. of injected oocytes & 12 & 24 & 24 & 30 & 30 & 120 \\
\hline With sperm deposited & 11 & 21 & 22 & 26 & 28 & 108 \\
\hline No. of survival & 10 & 19 & 20 & 23 & 26 & 98 \\
\hline Success rate (\%) & 91.7 & 87.5 & 91.7 & 86.7 & 93.3 & 90.0 \\
\hline Survival rate (\%) & 90.9 & 90.5 & 90.9 & 88.5 & 92.9 & 90.7 \\
\hline
\end{tabular}

failure occurred, the sperm were longer than average, causing the sperm tail tip to hang out of the micropipette. During oocyte penetration, the tail tip can adhere to the oocyte's ZP. After the micropipette was retracted out of the oocyte, the sperm came out of the cytoplasmic membrane of the oocyte with the micropipette. Aspirating the sperm further into the micropipette is not preferred because too large a volume deposited into an oocyte can negatively affect oocyte development.

The sample size tested in this study was not large. However, the preliminary results demonstrate that the robotic ICSI system is capable of dramatically reducing the learning curve of ICSI, capable of performing highly consistent ICSI operation, and is ready for large-scale animal/human trials.

\section{CONCLUSION}

This paper described the first robotic ICSI system capable of completing ICSI procedures that involve the manipulation of sperm and injection of oocytes. We developed a unique cell manipulation architecture by introducing a vacuum-based cell holding device for immobilizing many oocytes into a regular pattern, alleviating the challenge of random cell search. For sperm manipulation, a visual tracking algorithm was developed to track sperm head and low-contrast sperm tail for robotic immobilization of single sperm. Based on trials of 1000 sperm, $94.4 \%$ of the sperm were successfully immobilized by the robotic system. Furthermore, the robotic ICSI system performed complete ICSI procedures using the hamster oocyte-human sperm model. A success rate of $90.0 \%$ and a survival rate of $90.7 \%$ were achieved $(n=120)$. The robotic ICSI system demonstrated a high degree of reproducibility and operator skill independence. Clinical patient trial using the robotic ICSI system is underway.

\section{ACKNOWLEDGMENT}

The authors acknowledge financial support from the Natural Sciences and Engineering Research Council of Canada and Ontario Centres of Excellence.

\section{REFERENCES}

[1] G. Palermo, H. Joris, P. Devroey, and A. C. V. Steirteghem, "Pregnancies after intracytoplasmic injection of single spermatozoon into an oocyte," Lancet., vol. 340, no. 8810, pp. 17-18, 1992.

[2] T. F. Chan and L. A. Vese, "Active contours without edges," IEEE Trans. Image Process., vol. 10, no. 2, pp. 266-277, Feb. 2001.

[3] C. M. Bishop, Pattern Recognition and Machine Learning. New York: Springer-Verlag, 2006.

[4] Y. Sun and B. J. Nelson, "Biological cell injection using an autonomous microrobotic system," Int. J. Robot. Res., vol. 21, pp. 861-868, 2002.

[5] R. Kumar, A. Kapoor, and R. H. Taylor, "Preliminary experiments in robot/human cooperative microinjection," in Proc. IEEE Int. Conf. Intell. Robots Syst., Las Vegas, NV, 2003, pp. 3186-3191. 
[6] L. Mattos, E. Grant, R. Thresher, and K. Kluckman, "New developments towards automated blastocyst microinjections," in Proc. IEEE Int. Conf. Robot. Autom., Roma, Rome, 2007, pp. 1924-1929.

[7] X. Y. Liu, Z. Lu, and Y. Sun, "Orientation control of biological cells under inverted microscopy," IEEE/ASME Trans. Mechatron., vol. pp, no. 99, pp. 1-7, Aug. 2010.

[8] M. Subbarao and J. K. Tyan, "Selecting the optimal focus measure for autofausing and depth-from-focus," IEEE Trans. Pattern Anal. Mach. Intell., vol. 20, no. 8, pp. 864-870, Aug. 1998.

[9] G. Trummer, C. Kurzals, R. Gehring, and D. Leistner, "Next-generation proximity and position sensors," Sensors, vol. 21, no. 3, pp. 14-21, 2004

[10] Y. T. Shen, N. Xi, K. W. C. Lai, and W. J. Li, "A novel PVDF microforce/force rate sensor for practical applications in micromanipulation," Sens. Rev., vol. 24, no. 3, pp. 274-283, 2004.

[11] W. H. Wang, X. Y. Liu, and Y. Sun, "Contact detection in microrobotic manipulation," Int. J. Robot. Res., vol. 26, pp. 821-828, 2007.

[12] P. Vanderzwalmen, G. Bertin, B. Lejeune, M. Nijs, B. Vandamme, and R. Schoysman, "Two essential steps for a successful intracytoplasmic sperm injection: Injection of immobilized spermatozoa after rupture of the oolema," Hum. Reprod., vol. 11, no. 3, pp. 540-547, 1996.

[13] G. D. Palermo, P. N. Schlegel, L. T. Colombero, N. Zaninovic, F. Moy, and Z. Rosenwaks, "Aggressive sperm immobilization prior to intracytoplasmic sperm injection with immature spermatozoa improves fertilization and pregnancy rates," Hum. Reprod., vol. 11, no. 5, pp. 1023-1029, 1996.

[14] H. Oku, M. Ishikawa, N. Ogawa, K. Shiba, and M. Yoshida, "How to track spermatozoa using high-speed visual feedback," in Proc. Int. Conf. IEEE Eng. Med. Biol. Soc., Hiromasa, Japan, 2008, pp. 125-128.

[15] M. Berezansky, H. Greenspan, D. Cohen-Or, and O. Eitan, "Segmentation and tracking of human sperm cells using spatiotemporal representation and clustering," in Proc. SPIE-Int. Soc. Opt. Eng., 2007, vol. 6512, p. $65122 \mathrm{M}$.

[16] V. R. Nafisi, M. H. Moradi, and M. H. Nasr-Esfahani, "Sperm identification using elliptic model and tail detection," in Proc. 4th World Enformatika Conf., Istanbul, Turkey, 2005, pp. 205-207.

[17] World Health Organization, WHO Laboratory Manual for the Examination of Human Semen and Semen-Cervical Mucus Interaction, 4th ed. Cambridge, U.K.: Cambridge Univ. Press, 1999.

[18] M. Javed, N. Esfandiari, and R. F. Casper, "Failed fertilization after clinical intracytoplasmic sperm injection," in Reproduct. BioMed. Online, vol. 20, pp. 56-67, 2009

[19] X. Y. Liu and Y. Sun, "Automated mouse embryo injection moves toward practical use," in Proc. IEEE Int. Conf. Robot. Autom., Kobe, Japan, 2009, pp. 526-531.

[20] T. Uehara and R. Yanagimachi, "Microsurgical injection of spermatozoa into hamster oocytes with subsequent transformation of sperm nuclei into male pronuclei," Biol. Reproduct., vol. 15, pp. 467-470, 1976.

[21] A. Ahmadi and S. C. Ng, "Sperm head decondensation, pronuclear formation, cleavage and embryonic development following intracytoplasmic injection of mitochondria-damaged sperm in mammals," Zygote, vol. 5, pp. 247-253, 1997.

[22] O. G. Marina, I. L. Larry, and J. L. Dolores, "Human sperm microinjection into hamster oocytes: A new tool for training and evaluation of the technical proficiency of intracytoplasmic sperm injection," Fertility Sterility, vol. 73, pp. 395-401, 2000 .

[23] P. Xia, "Intracytoplasmic sperm injection: Correlation of oocyte grade based on polar body, perivitelline space and cytoplasmic inclusions with fertilization rate and embryo quality," Hum. Reprod., vol. 12, no. 8, pp. 1750-1755, 1997.

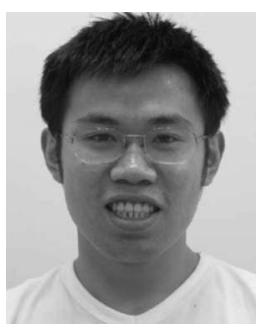

Zhe Lu (M'10) received the B.Eng. degree in control engineering from Harbin Institute of Technology, Harbin, China, in 2002, and the Ph.D. degree in mechanical engineering from the National University of Singapore, Singapore, in 2007.

$\mathrm{He}$ is currently a Postdoctoral Fellow in the Advanced Micro and Nanosystems Laboratory, Mechanical and Industrial Engineering Department, University of Toronto, Toronto, ON, Canada. His research interests include robotics, automation, and mechatronics.

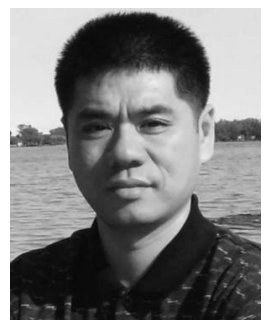

Xuping Zhang (M'11) received the B.Eng. degree in mechanical engineering from Chongqing University, Chongqing, China, in 1992, and the Ph.D. degree in mechanical engineering from the University of Toronto, Toronto, ON, Canada, in 2009.

$\mathrm{He}$ is currently a Postdoctoral Fellow in the Advanced Micro and Nanosystems Laboratory, Mechanical and Industrial Engineering Department, University of Toronto. His research interests include robotics, dynamics and control, vibration theory, and mechanisms.

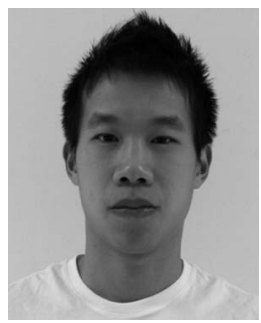

Clement Leung received the B.A.Sc. degree in electrical engineering from the University of British Columbia, Vancouver, Canada, in 2009. He is working toward the Master's degree in the Electrical and Computer Engineering Department at the University of Toronto, Toronto, ON, Canada.

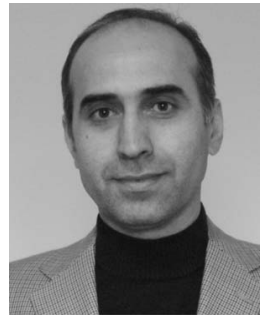

Navid Esfandiari received the residency training and the Ph.D. degree in medical immunology at Tehran University School of Medicine, Tehran, Iran, in 1994.

He was an Assistant Professor of immunology in Tehran, from 1994 to 2000. His fellowship was in andrology and infertility from the Cleveland Clinic, Cleveland, $\mathrm{OH}$, from 2000 to 2002 . He is a board certified High Complexity Clinical Laboratory Director (HCLD), certified by the American Board of Bioanalysis. He is Director of the ART Laboratories at TCART, and adjunct Professor in the Department of Obstetrics and Gynecology at the University of Toronto, Toronto, ON, Canada, and at the University of Tehran, Iran.

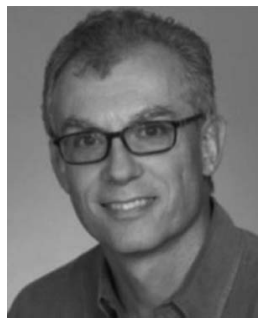

Robert F. Casper received the M.D. degree from the University of Western Ontario, London, ON, in 1973.

$\mathrm{He}$ is the Camille Dan Family Research Chair in Translational Cell Biology and a Senior Investigator in the Samuel Lunenfeld Research Institute at Mount Sinai Hospital. He is also a Professor of reproductive endocrinology and infertility in the Department of Obstetrics and Gynecology at the University of Toronto, ON, Canada, with cross-appointments in the Departments of Physiology, Medicine (Division of Endocrinology), The Institute of Medical Sciences, and in the Postgraduate Department of the Faculty of Dentistry at the University of Toronto.

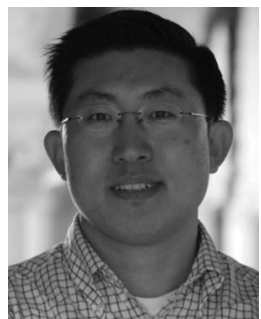

Yu Sun (M'03-SM'07) received the Ph.D. degree in mechanical engineering from the University of Minnesota, Minneapolis, MN, in 2003.

$\mathrm{He}$ joined the University of Toronto, ON, Canada, in July 2004, where he is is an Associate Professor now. Earlier he was a research scientist at the Swiss Federal Institute of Technology (ETH-Zrich). His research interest includes design and fabrication of MEMS/NEMS devices; micronanorobotic manipulation under optical and electron microscopes; and manipulation and characterization of biological cells, biomolecules, and nanomaterials using robotics, and MEMS/NEMS/microfluidics approaches. 